\title{
Posture tracking of multiple individual fish for behavioral monitoring with visual sensors
}

Article in Ecological Informatics · July 2016

DOI: 10.1016/j.ecoinf.2016.07.004

CITATIONS

0

5 authors, including:

Chunlei Xia

Pusan National University

21 PUBLICATIONS 71 CITATIONS

SEE PROFILE
READS

61

Some of the authors of this publication are also working on these related projects:

Project

A study on three-dimensional monitoring of individual behavior in fish group based on stereo vision View project

Developing greenhouse robots for pest sampling and control with minimal dose of pesticide View

Project project 


\title{
Posture tracking of multiple individual fish for behavioral monitoring with visual sensors
}

\author{
Chunlei Xia ${ }^{\mathrm{a}, \mathrm{b}}$, Tae-Soo Chon ${ }^{\mathrm{c}}$, Yuedan Liu ${ }^{\mathrm{d}}$, Jing Chi ${ }^{\mathrm{e}}$, JangMyung Lee ${ }^{\mathrm{b}, *}$

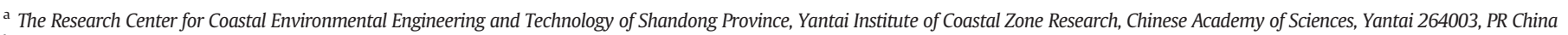 \\ b School of Electrical Engineering, Pusan National University, Busan, Pusan, 609-735, Republic of Korea \\ c Department of Biological Sciences, Pusan National University, Busan, Pusan, 609-735, Republic of Korea \\ d The Key Laboratory of Water and Air Pollution Control of Guangdong Province, South China Institute of Environmental Sciences, MEP, Guangzhou 510065, PR China \\ e Yantai Center, Shandong Technology Transfer Center, Chinese Academy of Sciences, Yantai, 264003, PR China
}

\section{A R T I C L E I N F O}

\section{Article history:}

Received 8 January 2015

Received in revised form 7 July 2016

Accepted 12 July 2016

Available online $\mathrm{xxxx}$

\section{Keywords:}

Fish pose measurement

Head and tail identification

Movement patterns

Fish aggregation

Behavioral analysis

Behavioral sensing

\begin{abstract}
A B S T R A C T
A novel scheme for the behavioral monitoring of multiple fish is presented based on measurements of the fish posture. Image identification of the fish head and tail is proposed by examining the gray level appearance features. A simplified posture of fish was calculated from its head and centroid. Multiple individual tracking was developed by incorporating the fish posture and global nearest neighbor. The proposed tracking scheme was tested with 2-5 individual Zebra fish (Cyprinidae, Danio rerio) under laboratory conditions. The experimental results confirmed the stability of the identification of a fish head and tail and a robust tracking performance was achieved. The proposed method could accurately record the movement trajectories and posture information of each individual. The tracking errors were only $0.278 \%-1.572 \%$ for $2-5$ individual fish while the posture measurement error rates were less than $0.16 \%$. Furthermore, the movement patterns of the fish, e.g., angular variations and distribution of the movement angle, were investigated from the behavioral data, which revealed the advantages of the proposed posture measurement for behavioral studies. The aggregation behavior of multiple fish was also analyzed to reveal the interaction patterns of the individuals in response to the spatial formation of the neighbors.
\end{abstract}

C 2016 Elsevier B.V. All rights reserved.

\section{Introduction}

Behavioral changes reflect the accumulation effects of disturbances or stress from the environment. Behavioral monitoring is an efficient and fundamental tool for a long-term study of aquatic ecosystems and water quality assessments, which are more ecologically relevant and economical than chemical sensors (Gerhardt, 1999; Park et al., 2005; Bae and Park, 2014). Fish as a biological indicator have been used widely in biological monitoring systems for environmental studies owing to their sensitivity to changes in the environmental parameters (Chon et al., 2005; Gerlai et al., 2006; Masud et al., 2005; Ren and Wang, 2010).

Biological early warning systems have shown great progress over the last decade since more advanced technologies were developed and integrated to a behavioral monitoring system (Bae and Park, 2014). With the vast improvements in the computational capability of personal computers and the implementation of reliable image processing techniques, the visual monitoring of fish behavior has becomes a common tool for biological and environmental studies, e.g., exploiting

\footnotetext{
* Corresponding author.

E-mail addresses: c.xia2009@gmail.com (C. Xia), tschon@pusan.ac.kr (T.-S. Chon), jmlee@pusan.ac.kr (J. Lee).
}

the fish behavior for environmental risk assessments and their responses under specific stimulation, such as chemical stress analysis (Barry, 2012; Kane et al., 2004; Ma et al., 2010). A number of video surveillance systems have been developed for automatic observations and measurements of fish behavior since the 1990s (Delcourt et al., 2013; Kato et al., 1996; Kuklina et al., 2013; Papadakis et al., 2012; Suzuki et al., 2003). One of the major advantages of an automatic behavioral monitoring system is that no human interference is required during the observation process. Long-term monitoring is possible using an automatic observation system, in which the behavioral monitoring can be conducted for hours, days, months or even longer. In addition, the behavioral data could be quantified by the observation program for computational analysis, which is almost impossible by human inspection.

In the early stages, the EthoVision system was developed for automated observations of animal behavior and was applied to observe fish behavior (Delcourt et al., 2006; Noldus et al., 2001). Kato et al. (2004) presented an observation system dealing with the behavioral measurements of two fish. The chasing behavior of two fish was defined by examining the distance and angle between them. The interaction between two fish was also discussed through experiments. A multitracking system was developed to record the movement trajectories of multiple fish (Delcourt et al., 2006; Delcourt et al., 2009). The 
multitracking system tracked the fish movements by predicting the theoretical position of each individual and matched to the nearest neighbor of the target fish (Delcourt et al., 2009). A Kalman filter and particle filter are the most widely used techniques for predicting the positions of moving targets for tracking tasks, including the movement tracking of multiple fish (Fontaine et al., 2008; Pinkiewicz et al., 2008; ). Recently, more sophisticated tracking techniques have been developed to improve the performance of behavioral analysis of fish and the maximal number of targets is up to 50 individuals for 2D tracking (Branson et al., 2009; Papadakis et al., 2012). The tracking accuracy of multiple fish from an occlusion is enhanced greatly by the "fingerprint" technique, which identifies each individual fish by their appearance (Pérez-Escudero et al., 2014).

Previous studies usually treated an individual fish as a single point in two- or three-dimensional space, either for developing a behavioral tracking system or for behavioral analysis. Most current behavioral observation programs identify an individual fish as a single image blob and its movement trajectory is produced only by measuring the centroid of the fish. On the other hand, posture analysis for fish has not been studied widely. Posture analysis represents more precise behavioral information regarding the specific stimulation and environmental changes.

Most existing fish tracking systems could not examine fish poses during the observations and it was almost impossible to determine the detailed status of the fish, such as angular variations, body directions or body shaking. On the other hand, integrating the fish posture could improve the stability of multiple fish tracking, particularly when fish aggregate. This paper presents a multiple individual tracking scheme by integrating the fish posture and investigate the behavioral pattern of fish by analyzing the posture information. This study developed a posture measurement method based on the image identification of fish head and tail. The gray level appearance features were employed to identify the fish head and tail. The proposed method was effective in low resolution images and was computational inexpensive. A simplified fish posture was represented by an angle formed by linking the fish head and centroid. Subsequently, the fish posture was integrated to the global nearest neighbor for tracking multiple individual fish. Robust tracking performance was achieved using the proposed tracking scheme. Subsequently, posture analysis was conducted further based on the behavioral data from the observation experiments, and the aggregation movement of multiple fish was also discussed.

\section{Materials}

Fig. 1 presents the structure of a two-dimensional behavioral monitoring system. This observation system consists of an aquarium, digital camera, light source and personal computer. The behavioral observations were conducted in a $300 \times 300 \times 300 \mathrm{~mm}^{3}$ glass aquarium and a

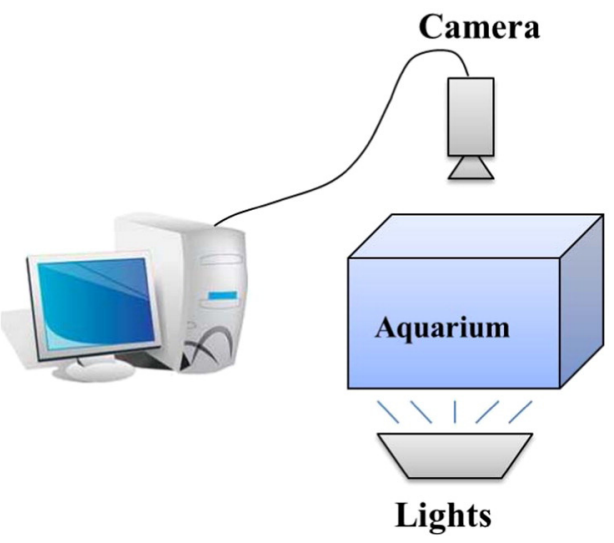

Fig. 1. Structure of the observation system. webcam (Logitech ${ }^{\circledR}$ C905) was placed approximately $20 \mathrm{~cm}$ above the aquarium. As fish always have a cone-shape appearance in the top view of an aquarium; measuring the posture of the fish and tracking the movements of multiple fish from the top view could be stable and reliable. In addition, the field of view of the camera should cover the whole arena of fish by adjusting the camera height. A common white light was installed under the aquarium to achieve the optimized contrast between the fish and the aquarium. Consequently, a uniform illumination condition was constructed while reflections on the water surface and shadows were avoided. The proposed tracking algorithm was implemented using Microsoft Visual $\mathrm{C}++10.0$ and an open source computer vision library (OpenCV) on a personal computer (Intel ${ }^{\circledR}$ Core $^{\mathrm{TM}} 2$ Duo CPU E4500@ $2.20 \mathrm{GHz}$ with 2 G memory).

Zebra fish, Danio rerio, was chosen as the test species because zebra fish have been applied widely as an indicator in biological monitoring regarding biological information and vulnerability to chemical stress (Swain et al., 2004). The individual zebra fish were obtained from a local fish dealer. Adult zebra fish, approximately $3 \mathrm{~cm}$ in length, were selected in this work.

To demonstrate the performance of the proposed tracking scheme, the water depth was set to $3 \mathrm{~cm}$ to minimize the occlusions of fish. A low resolution video with $640 \times 480$ pixels was adopted by considering the storage space of video clips for long-term observations. Under this resolution, the zebra fish were approximate 30 pixels in length and 5 pixels in width. Video clips in 10-min lengths were tapped for the observations of $2-5$ individual fish. These video clips were used to evaluate the tracking performance and behavioral analysis.

\section{Behavioral tracking of multiple fish}

\subsection{Automatic initialization}

Fig. 2(a) presents captured aquarium image with multiple fish. An automatic initialization is presented to automatically detect the individual fish from the captured images and assign movement tracks to each individual. The detection of individual fish was conducted using the adaptive thresholding algorithm (Sauvola and Pietikainen, 2000). Adaptive thresholding is a local thresholding algorithm that is more robust to

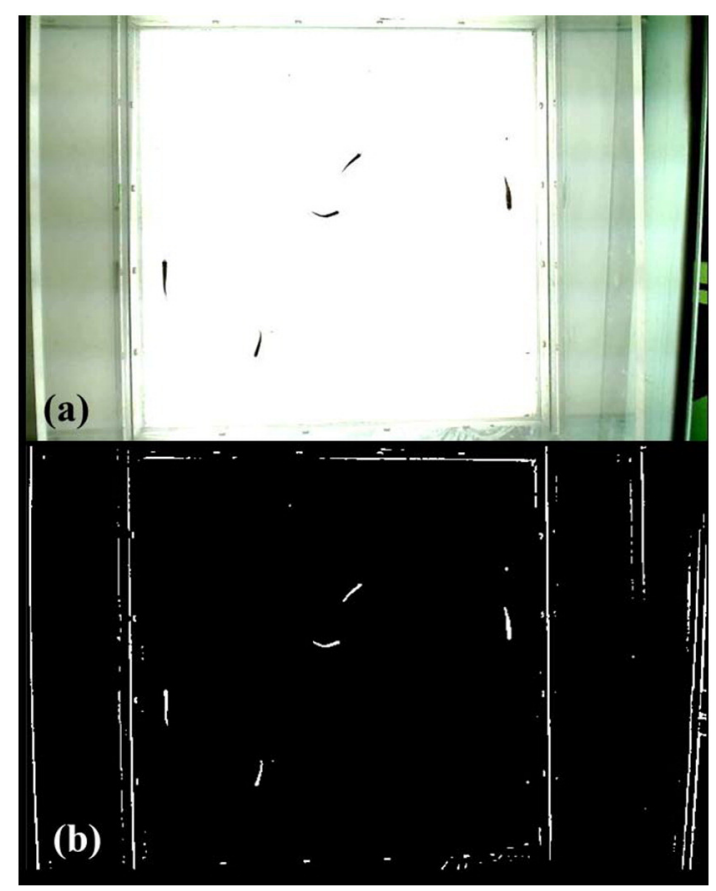

Fig. 2. Fish observations and segmentation, (a) top view of aquarium, and (b) binarized image by adaptive thresholding. 
the illumination changes compared to the widely used background subtraction method. Background subtraction requires calculating the mean image of the background, such as a mixture of Gaussians (Harville, 2002), to detect the motion of objects under varying light conditions. On the other hand, when a fish remains for a certain period in an aquarium (e.g., sleeping), the fish image would be modelled as a part of the background and the static fish could not be extracted from the background. Adaptive thresholding is more effective in segmenting the fish than the global thresholding method (e.g., Otsu segmentation) because adaptive thresholding estimates the threshold for each pixel from its neighboring area. Accordingly, individual fish were segmented as small blobs in the binarized image, as shown in Fig. 2(b). The small size image noises produced in the segmentation process were eliminated by applying Gaussian filtering and morphological operations (e.g., dilation and erosion).

Individual fish were determined by examining the shape features (i.e., height, width, and area) of the binary blobs. The blobs with the appropriate height and width were identified as a candidate of the fish. The ranges of height and the width of fish were determined by pretests. The width of each fish was 3-10 pixels and the height of a fish ranged from 20 to 40 pixels as the criteria for determining an individual fish.

\subsection{Fish posture measurement}

In this paper, measurements of the fish posture are proposed to calculate a simplified fish pose by identifying the head and tail of fish. The fish posture is described by a global angle $\alpha$, which was calculated from only the head $\left(C_{h}\right)$ and centroid of the fish $(C)$, as illustrated in Fig. 3a and $\mathrm{b}$. The posture angle, $\alpha$, was valued in the range, $[0,2 \pi]$, to express global movement direction of the fish. The fish posture also indicated the movement direction of an individual fish.

Measurements of the fish posture are dependent on the accurate identification of a fish head and tail. This is because the images of fish were taken from the top view and the fish body is presented in a cone shape or bended cone shape. The individual fish was then bordered by

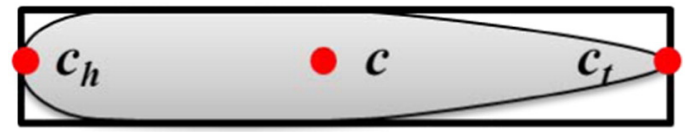

(a)
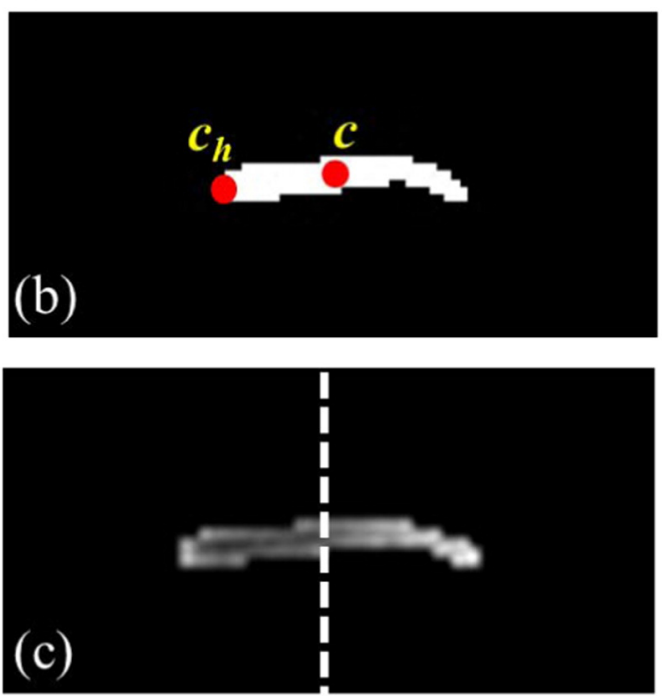

Fig. 3. Scheme for determining the fish head and tail area, (a) definition of the head and tail, (b) markers of the head and centroid of a binary fish image and (c) gray level appearance of a fish head and tail. the minimum bounding box. The centroid of the fish was obtained by the gravity center of a binary fish image while the fish head and tail are described by the center points on the short sides of the minimum bounding box (Fig. 3a). To distinguish the head and tail, the fish image is divided equally into two parts to represent the head and tail of a fish (Fig. 3c). The fish head shows a broad round shape ("U" shape), whereas the tail part shows a sharpened tip ("V" shape). Because the fish head and tail parts have very different shapes, examining the curvatures might be efficient in identifying the fish head and tail. On the other hand, the image resolution was low $(640 \times 480$ pixels $)$ in that the head and tail areas were too small. The geometric features of the head and tail parts showed no difference under a low image resolution (Fig. 3b).

The gray level histogram revealed significant differences in the fish head and tail images. The fish head and tail can be identified according to their appearance features. The appearance of the head part shows a dark tone while the tail part contains brighter pixels (Fig. 3c). Because the fish tail is thin and the tail fin is translucent, light diffusion occurred in the image of the fish tail and contributed to the bright appearance. By analyzing the gray level histogram of the fish head and tail area, most pixels of the head part was smaller than 150 and the tail part contained more pixels larger than 150 in gray intensity. For simplification, the mean intensities of the head and tail area were examined to determine the head and tail because the tail part showed a higher mean intensity value than the head part. This method was computationally inexpensive and extremely stable in the identification tests.

\subsection{Multiple individual tracking}

Individual fish and their postures were measured according to the previous steps. At the current step, the task of multiple tracking is to find the optimal pair matching for all individuals from time, $t-1$, to time $t$. All possible candidate pairs for obtaining the movement trajectory are connected by dashed lines in Fig. 4(a). A global nearest neighbor (GNN) was devised with the fish posture to solve the data association issue for tracking multiple individuals (Cai et al., 2006; Konstantinova et al., 2003). The symbol $f_{i}^{t-1}$ and $f_{i}^{t}$ indicate the $i$ th individual fish at time $t-1$ and time $t$, respectively.

Initially, gating is given to reduce the calculation of the irrelevant measurements at time $t$. The tracking gate is restricted by the maximal movement distance $\left(d_{T}\right)$ and movement angle $\left(\alpha_{T}\right)$ (Fig. 4b). The maximal movement distance is the greatest distance that a fish can swim within a short time period, $\Delta \mathrm{t}$ (e.g., $0.033 \mathrm{~s}$ ). The movement angle is the angle of the tracking gate that indicate the maximal turning angle of a fish within a small time period. The maximal movement distance and movement angle form a sector of finding the candidates for tracking. The values of the maximal movement distance and movement angle were given according to the authors' experience and were estimated from pre-tests. The value of $d_{T}$ was set to 100 pixels for an image resolution of $640 \times 480$ pixels and the angle, $\alpha_{T}$, was determined to be $50^{\circ}$. Furthermore, the posture difference $\left(\alpha_{i}\right)$ was examined to remove the candidates in the tracking gate with an improper movement direction. The posture difference is the absolute value of the angular difference of two individuals. A large posture value indicates two fish moving from different directions and should not be paired in the movement trajectory. For example, the face to face movement of two fish could not be confused by checking the posture difference. The incorrect individual tracking from the aggregation movement could be reduced strongly by checking the posture difference. The posture difference was limited to $90^{\circ}$ in this study.

Subsequently, these tracking parameters were utilized as the constraints to find the minimal cost between $f_{i}^{t-1}$ and $f_{i}^{t}$ using the GNN algorithm. To find the global nearest neighbor of the tracking candidates, a cost matrix $(C)$ was given to represent all the costs among $f_{i}^{t-1}$ and $f_{j}^{t}$ :

$C_{i j}\left(f_{i}^{t-1}, f_{j}^{t}\right)=d_{i j} \lambda_{i j}$, 


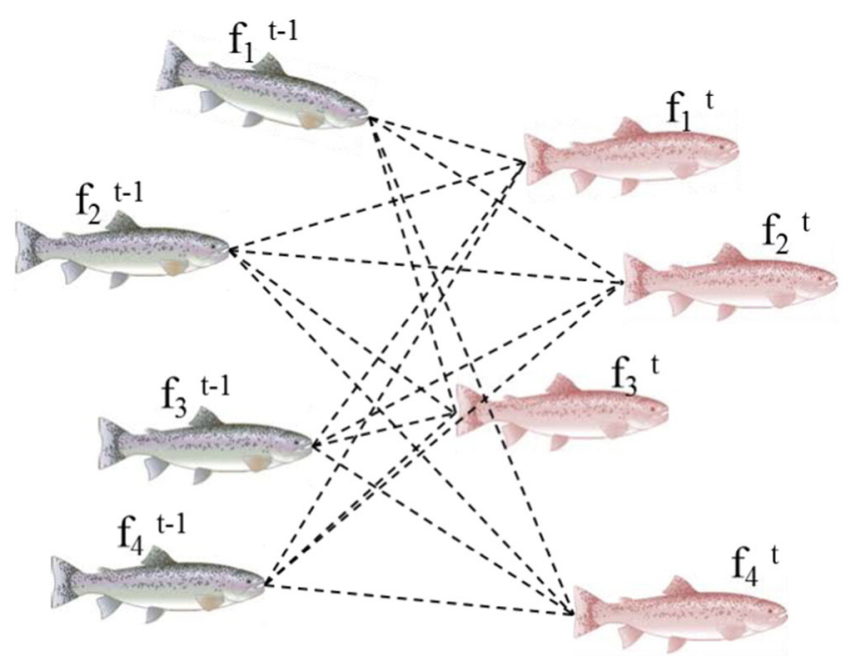

(a) time $t-1$

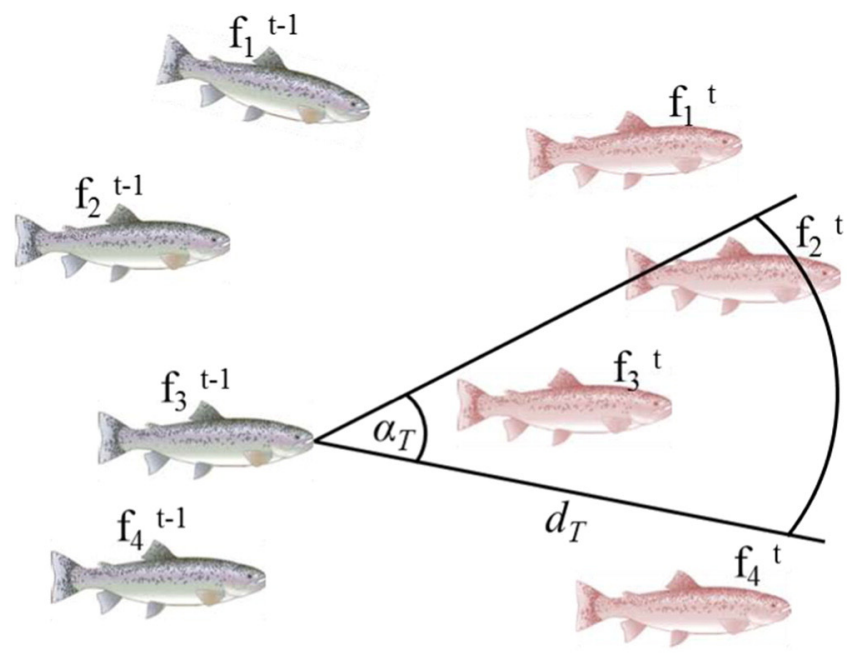

(b) time $t-1$

time $t$

Fig. 4. Strategy of tracking multiple individuals, (a) all possible associations and (b) constraints for pair matching.

$d_{i j}=\operatorname{dist}\left(f_{i}^{t-1}, f_{j}^{t}\right), i \in[1, n], j \in[1, m]$,

$\lambda_{i j}=\left\{\begin{array}{c}1, \text { if }\left|\alpha_{i}-\alpha_{j}\right|<\alpha_{T} \text { and } d_{i j}<d_{T}, \\ \infty, \text { otherwise }\end{array}\right.$

where $d_{i j}$ is the Euclidian distance of two fish and $\lambda_{i j}$ is the constraint for gating individual, $i$, at time $t$. The cost matrix $(C)$ consists of $n \times m$ elements. The number of tracking targets $(n)$ is a constant that is determined at the initialization stage of tracking, and $m$ is the number of actual measurements at time $t$. In addition, obtaining the optimal pairs is expressed as a linear assignment problem:

$\min \sum C\left(f_{i}^{t-1}, f_{j}^{t}\right)$

The Hungarian algorithm was applied to optimize the pairing of individuals from time $t-1$ to time $t$, and the movement trajectories were assigned for each individual.
Because the occlusions and failure to detect the fish lead to losses, tracking the miss-detected individuals may not be paired in this case. If an individual from time $t-1$ cannot be paired, this individual should maintain the position at time $t$ and should be paired in the next frame. At each frame, the pairing process is performed iteratively until no more candidates can be paired. In addition, the searching range, $d_{T}$, and movement angle, $\alpha_{T}$, are increased at each iteration to pair more candidates.

\section{Results \& behavioral analysis}

\subsection{Tracking performance}

Multiple fish tracking was tested with the video clips of multiple individuals. Fish behavioral observations were conducted using the developed multiple tracking program (Fig. 5). The parameters were tuned to optimize the tracking performance. Fig. 5(a) presents the real-time image captured from the top view of an aquarium with tracking results. The head and body centroid of each fish were marked with color dots to distinguish each individual. The numbers located in the top-left of the aquarium image was the sequence number of the video frame (Fig. 5a). The movement trajectories were obtained in real-time from the tracking results and Fig. 5(b) shows the movement trajectories of 5 individuals.

The original outputs of the behavioral observation were the twodimensional $x-y$ coordinates of the fish head and centroid, which was recorded frame by frame in the plain text files. The movement trajectory can be regenerated easily with these time-series point data and the computational methods could be applied for further behavioral analysis, such as a Self-Organizing Map, HMM (Liu et al., 2011; Wang et al., 2014).

The processing time of multiple fish tracking in each frame was less than $0.033 \mathrm{~s}$ (i.e., $30 \mathrm{fps}$ ), in which the real-time tracking was achieved in this work. Two modes of multiple tracking were available in the proposed tracking scheme: behavioral tracking could be carried out using either video clips or online observations from real-time cameras.

The tracking performance was evaluated by manually checking the automated identification and the ground truth frame by frame. The ability of multiple tracking was analyzed using 2-5 individuals. First, the occlusion rates and tracking accuracy from occlusions are presented in Fig. 6. In this study, the occlusion of fish was defined as the overlap among fish in the vertical direction or the image of the attached fish, which the caused failure of segmentation of individuals. Exchanging the movement tracks of the fish after the occlusion is a common issue in the multiple individual monitoring systems (Delcourt et al., 2009). As the water depth was limited to $3 \mathrm{~cm}$, overlapping among fish seldom occurred in the observations while the attaching of several fish produced more difficulties for individual tracking. As concluded from the experiments, the occlusions did not appear frequently during the behavioral observations and the proposed tracker showed rather low rates of incorrect tracking of multiple individuals. The occlusions of
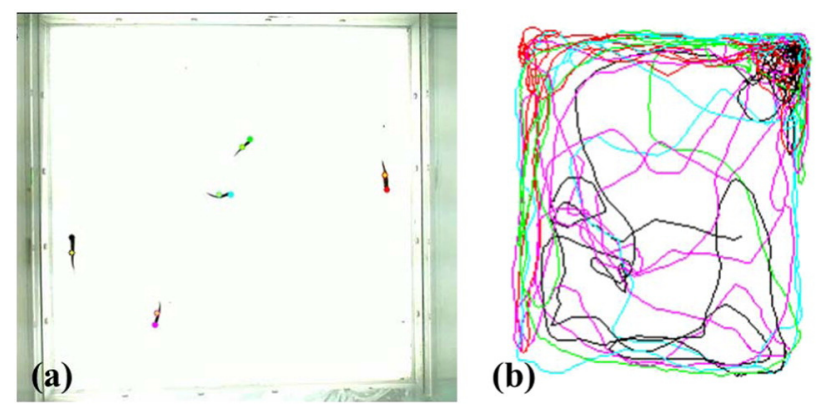

Fig. 5. Behavioral tracking of multiple fish, (a) individuals with tracking makers and (b) their movement trajectories. 


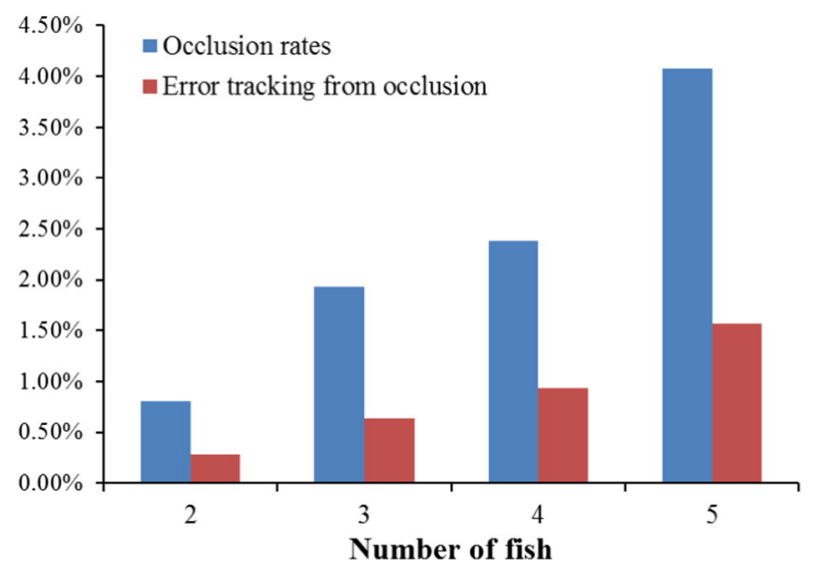

Fig. 6. Occlusion rates and error tracking from occlusions.

fish were investigated and are illustrated in Fig. 6, which shows occlusion rates out of the total video frames $(18,000$ frames in total) of $0.806 \%, 1.933 \%, 2.383 \%$, and $4.072 \%$ for $2-5$ individuals, respectively. Accordingly, the incorrect tracking was examined and the corresponding rates of exchange of the tracks for the $2-5$ individuals during the observations were only $0.278 \%, 0.639 \%, 0.939 \%$, and $1.572 \%$.

The error tracking rates from the occlusion were significantly lower than the occlusion rates, which indicate the proposed tracking method performed stably against the occlusions of the fish. The trackers had a high probability of following the fish correctly after occlusion because the movement direction of the fish improved the accuracy of the associating individuals. On the other hand, the occlusions of the fish occurred for a short period, in which the occlusion duration was varied from 2 to 19 frames during the observations.

The performance of multiple individual tracking from the occlusion is presented in Fig. 7. The black line shows the number of occlusions occurred in the observations. The number of error tracking, i.e., exchange of movement tracks, is drawn in the colored bars. The total number of error tracking was $5,12,18$, and 30 for the observations of $2-5$ individuals, respectively. The error tracking was due mainly to occlusions but only a small proportion of occlusions caused incorrect fish tracking (Fig. 7). A few tracking errors occurred without occlusions. Tracking errors without occlusion were not observed in the 2-individual observations. In addition, the exchange of movement tracks without an occlusion only occurred once for the 3-and 4-individual observations and 3 times of exchange was observed in the 5-individual observations.

Fig. 8 presents the total number of incorrect recognition of fish head and tail. The identification errors were 3, 4, 6, and 28 with 2-5

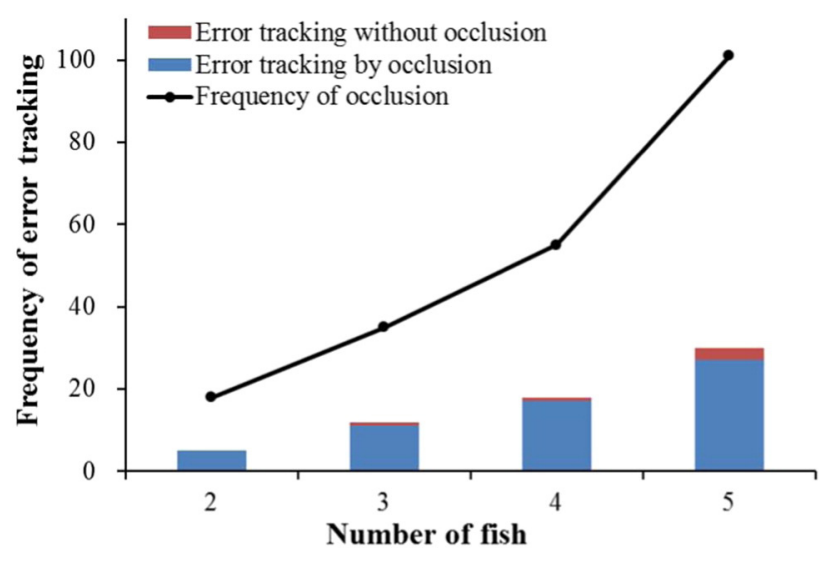

Fig. 7. Frequency of occlusion and tracking errors.

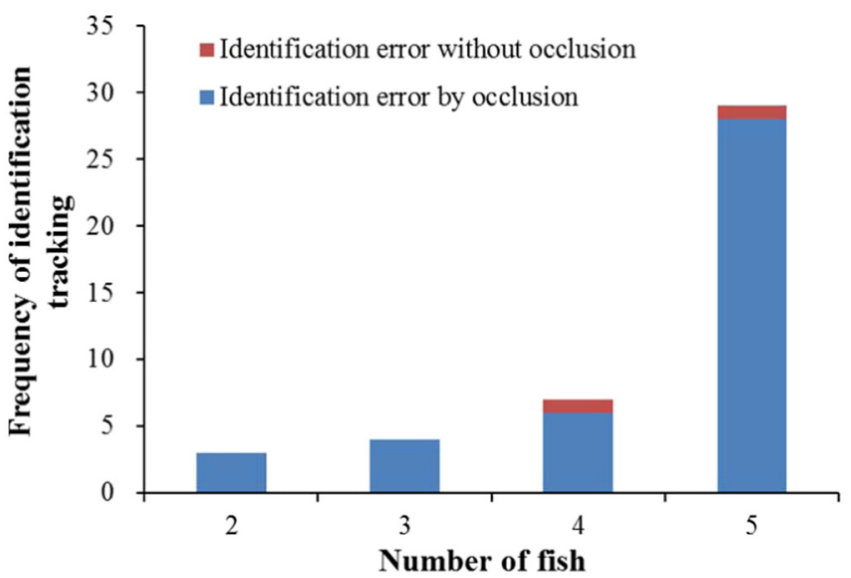

Fig. 8. Identification errors on fish head and tail.

individuals that equaled $0.016 \%, 0.022 \%, 0.033 \%$, and $0.16 \%$ from the overall observation period. The incorrect identification rarely occurred without occlusions. The identification errors without occlusions only appeared once for the 4-individual and 5-individual observations and the duration of incorrect identification were 5 and 17 frames, respectively. The occlusion was the main reason for the identification errors on the fish head and tail. On the other hand, the individual fish were barely segmented from the occluded fish images, and the proposed identification algorithm was unable to examine the head and tail in this situation. The observation experiments showed that the proposed identification method was extremely stable for distinguishing head and tail of a single fish.

\subsection{Preliminary analysis of fish movements}

Preliminary analysis of the observed behavioral data was conducted to show the behavioral patterns of the fish. Angular variations and the overall movement pattern of the fish were examined from the observed results. Subsequently, the aggregation behavior of the multiple individuals was investigated to reveal the interactions of the fish.

The angular variation was defined as the angular difference in the same individual between at time $t-1$ and time $t$. The angular variation was the rotational degree of an individual fish within a small duration, $\Delta t$. The angular variation represents the speed of the directional changes of an individual fish, e.g., a sudden directional change, body shaking or self-turning behaviors could be investigated by examining the angular variations. Because the posture of fish was available in this study, accurate angular variations could be calculated from the tracking results. The angular variations within $0.1 \mathrm{~s}$ and $0.5 \mathrm{~s}$ were obtained from 5 - individual observations (Fig. 9). The angular variations were calculated for each individual and the overall distribution pattern of angular variation is presented in Fig. 9.

Within a short time interval $(0.1 \mathrm{~s})$, the angular change was less than $20^{\circ}$, which suggests that fish moved smoothly and sudden directional changes were not occurred frequently (Fig. 9a). The proportion of large directional changes in the body angle was presented when the time interval was increased to $0.5 \mathrm{~s}$ (Fig. 9b). Because the fish had more movements within a longer time duration $(0.5 \mathrm{~s})$, the accumulation of angular changes increased the proportion of large angular variations. On the other hand, most of the angular variations were valued at less than $20^{\circ}$, which indicates that the fish moved smoothly along the same direction. The angular variations could provide a precise description of fish movement and could be novel criteria for studying the behavioral response. The normal pattern of angular variations should be studied for a biological warning system in aquatic ecosystems, which is beyond the scope of this study. 
$0.1 \mathrm{~s}$
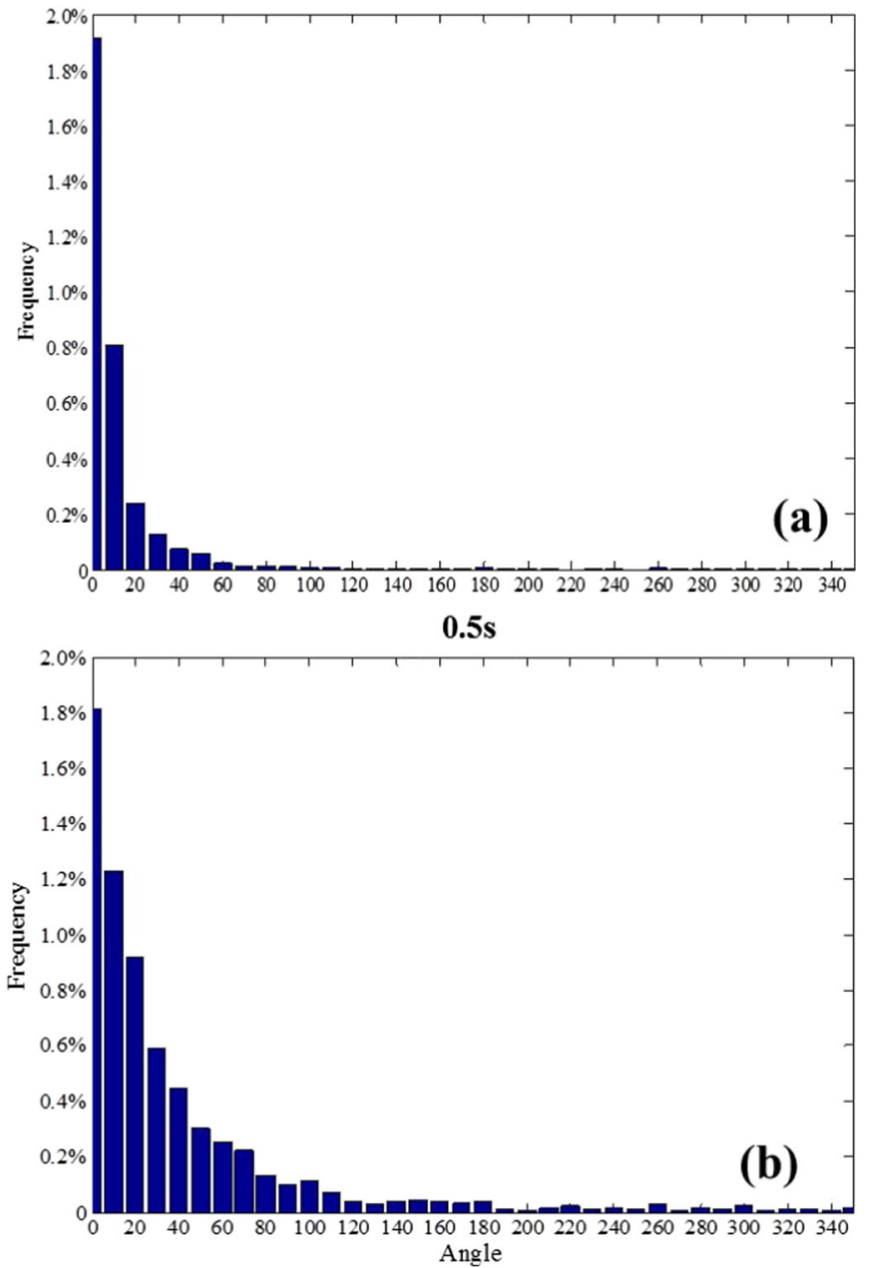

Fig. 9. Angular variations within $0.1 \mathrm{~s}$ and $0.5 \mathrm{~s}$.

Subsequently, the overall distribution pattern of the fish posture was obtained by computing the distribution of body angles from the observation duration. The overall distribution pattern of the fish posture indicated the trend of movement for one or a group of individuals. The posture angle ranged from 0 to $360^{\circ}$. Fig. 10(a) showed the posture pattern of the two-individual observation while Fig. 10(b) shows the posture distribution of 5 fish. Periodic wave patterns appeared in the distribution of postures (Fig. 10). In addition, periodic peaks in these distributions were located around $0,90,180$, and $270^{\circ}$ with a similar frequency. These periodic posture patterns might be caused by the boundary effect, in which fish preferred to move along the boundary of the aquarium. Because a rectangular aquarium was used as the observation arena and fish moved frequently along the boundaries, which contributed a large proportion of vertical and horizontal movements. Therefore, four peaks of vertical and horizontal movements were observed in the movement patterns. The peaks showed similar frequencies, which indicate that the movement of fish was distributed randomly along the boundary.

In addition, the interactions among the individual fish in the group were investigated. Aggregation analysis indicated the frequency of several fish active within a certain area (Fig. 11). The proportion of fish aggregation out of the overall observation period was calculated with an increasing area of aggregation. For example, to analyze two-fish aggregation within an area with a diameter of 100 pixels, the total frames containing two fish located within 100 pixels were counted and its proportion of the overall observation period was obtained. Fig. 11 presents the diameter of the aggregation area in the $x$ axis; the maximal value of
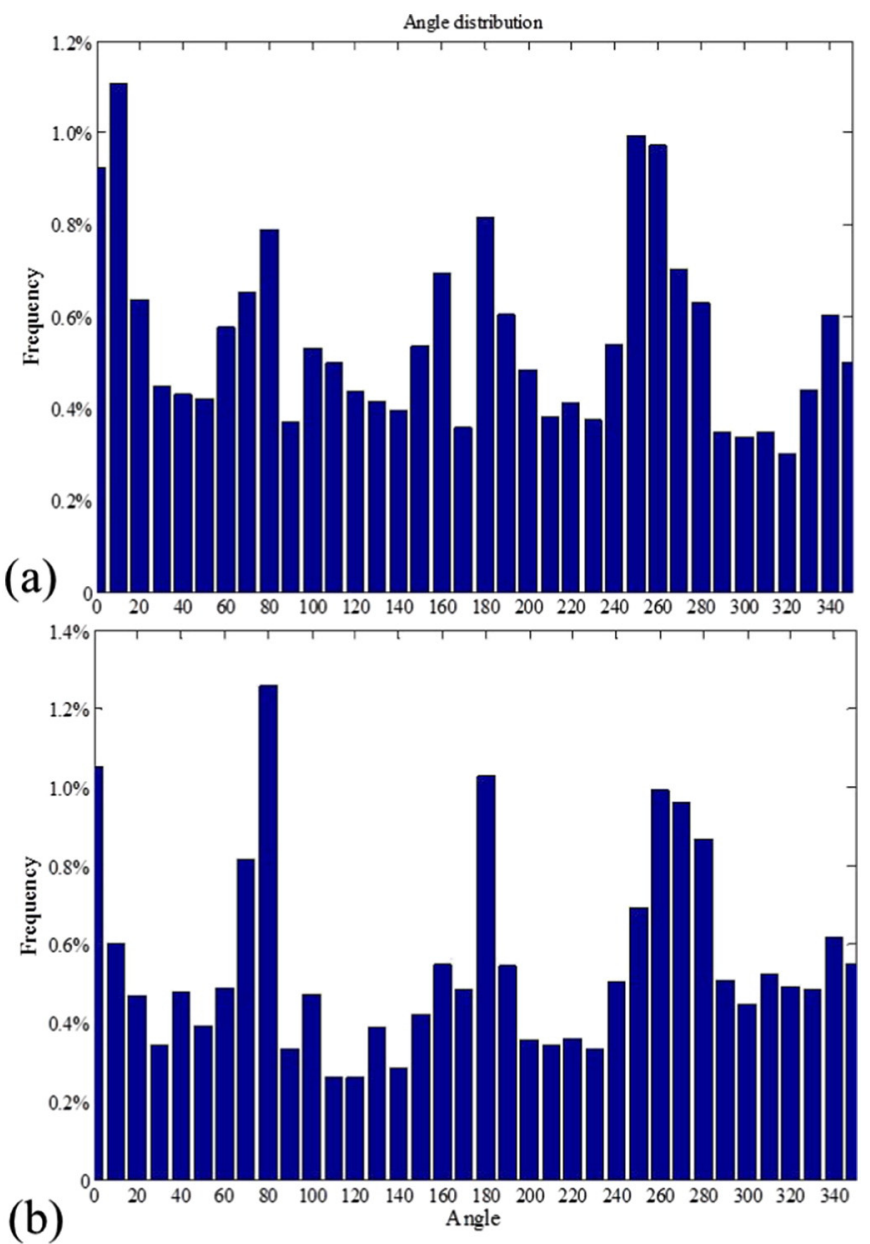

Fig. 10. Overall pattern of fish movement, (a) angle distribution of two-individual observations and (b) angle distribution of five-individual observations.

the $x$ axis was 450 pixels, which was the length of the diagonal of the arena.

The fish body had approximate 3-8 pixels in width and 20-40 pixels in length. The area size for analyzing the aggregation of multiple fish was given from 10 to 410 pixels because the diagonal of the observation area is 410 pixels. Fig. 11 shows the fish aggregation from 2 to 5 individuals with an increasing area size. The fish group containing 2-5 individuals were examined with a different number of observation targets. The results indicated the active area of the fish, which increased with increasing number of targets. Fig. 11 shows the overall active area of 2-individual to 5-individual observations with increasing area size. For approximately $90 \%$ of the duration, the fish moved within a round area with a diameter of 350 pixels when observing 3 , 4 , and 5 individuals, while the area of 230 pixels contained $90 \%$ activities for 2-individual observations (Fig. 10). The interactive behaviors of two fish increased with more individuals in the observation. In the 2 -individual observations, only $10.5 \%$ of the duration that two-fish aggregation was observed within a 30-pixel circle and $27.5 \%$ duration fish active was observed within a 50-pixel circle (Fig. 11a). While the twofish aggregation within a 30-pixel circle were 39.0\%, 33.2\%, and 39.6\% in 3-, 4-, and 6-individual observations, respectively. The proportions of two-fish aggregation within a 50-pixel circle were up to $69.3 \%$, $63.4 \%$, and $70.0 \%$ in 3-, 4-, and 6 -individual observations. The results showed that the proportion of two-fish aggregation increased dramatically with increasing area size from 20 to 70 pixels (Fig. 11). On the other hand, the aggregation of 3,4, and 5 individuals were not increased significantly, which inferred that the two-fish interaction was preferred. 

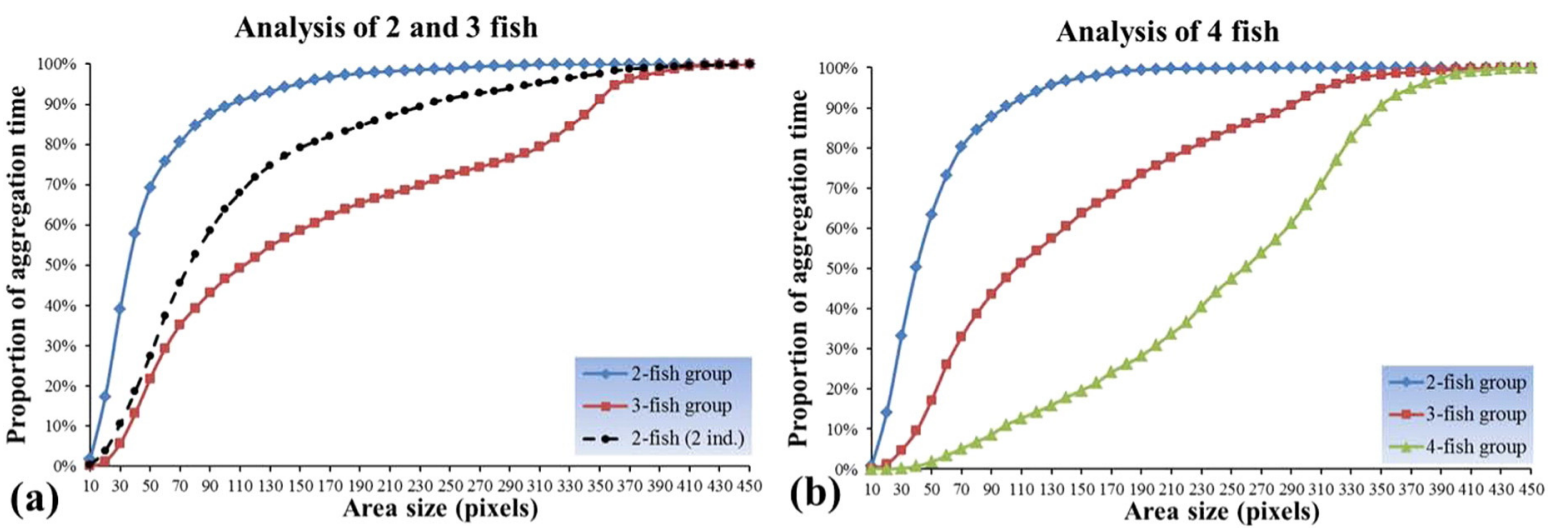

Analysis of 5 fish

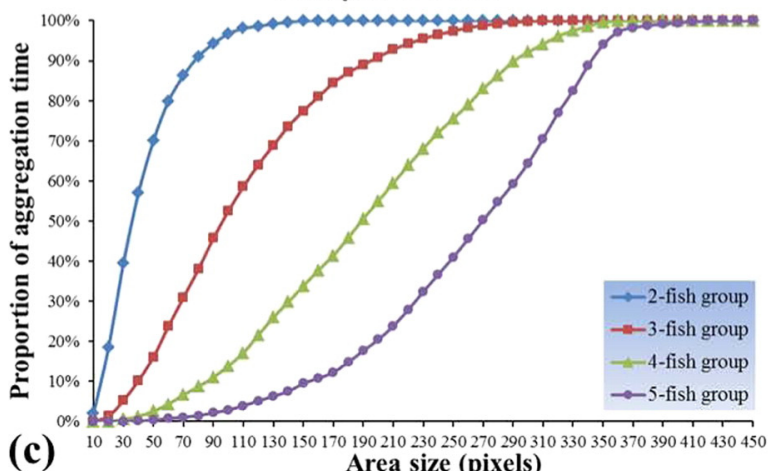

Area size (pixels)

Fig. 11. Aggregation of fish, (a), (b) and (c) proportion of aggregation of 2-5 individuals.

\section{Discussion}

An individual tracking scheme was proposed for behavioral observations of multiple fish by identifying the fish head and tail. The identification accuracy of the fish head and tail was outstanding and the stability of fish tracking was demonstrated through experiments on multiple individual observations. The proposed method was rather computationally inexpensive, which was suitable for real-time applications on low configuration hardware. The video resolution was only $640 \times 480$ pixels at $30 \mathrm{fps}$, a requirement of which any of the current webcam products could satisfy. The low image resolution is important for long term observations due to the limited storage space. A high resolution video could provide more detailed information on the fish and could improve the tracking performance; however, higher image resolution requires more than 10 times the storage space. At the current stage, a high resolution video is still unaffordable for recording the long term behavioral movements. The high resolution video could also increase the computational cost and requires a high performance computer to process the behavioral data. The low image resolution and lower computational cost have advantages for running on mobile devices for large scale deployments, e.g., large scale sensing network.

The accuracy of multiple fish tracking was improved significantly by applying the fish posture as the constraints for tracking. The fish posture was integrated to the nearest neighbor association to enhance the stability of tracking multiple individuals from the occlusion and aggregation of fish. The movement prediction algorithms, such as Kalman filter or particle filter, were unnecessary in the proposed tracking system. The trackers without posture constraints suffered from the difficulties of sudden directional changes, face to face movement, chasing behavior, and aggregation of fish (Fig. 12). The proposed method could track the individual movements accurately from the close interaction of the fish.

The face to face interaction of two fish is illustrated in Fig. 12 (a). Fish $\mathrm{A}$ and $\mathrm{B}$ at time $\mathrm{t}-1$ closely ran from the opposite direction and they moved to $\mathrm{A}^{\prime}$ and $\mathrm{B}^{\prime}$ at time t. Because the movement directions of the two fish are known in this work, the tracker could not confuse pair $A$ to $\mathrm{B}^{\prime}$ or $\mathrm{B}$ to $\mathrm{A}^{\prime}$.

Fig. 12(b) presents a typical case of two fish chasing is illustrated in which the two fish move closely in parallel and crossed to each other. Fish $\mathrm{A}$ and $\mathrm{B}$ moved to fish $\mathrm{A}^{\prime}$ and $\mathrm{B}^{\prime}$ at from time $\mathrm{t}-1$ to time $\mathrm{t}$; however, fish $\mathrm{A}^{\prime}$ and $\mathrm{B}^{\prime}$ both fell into the tracking gate of fish $\mathrm{A}$, and fish $\mathrm{B}^{\prime}$ was the nearest neighbor of fish $A$. In this situation, fish $B^{\prime}$ might be incorrectly paired with fish $A$ if only the nearest distance was considered. Fish $\mathrm{B}^{\prime}$ was the nearest neighbor for both fish $\mathrm{A}$ and $\mathrm{B}$; the optimal pairing was solved using the Hungarian algorithm. Fish B and fish $\mathrm{B}^{\prime}$ were paired because the bipartite nearest distances were found between fish B and $\mathrm{B}^{\prime}$. Therefore, the crossing movement of fish was

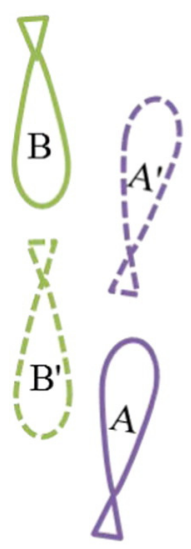

(a)

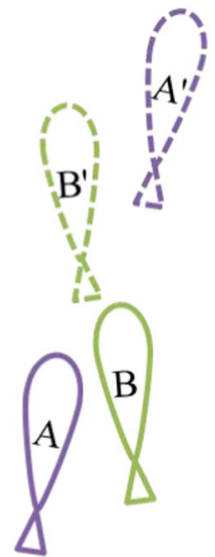

(b)

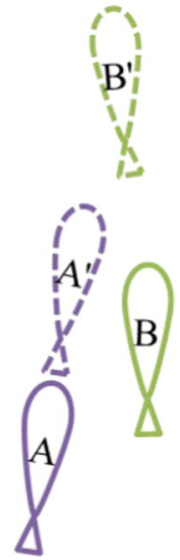

(c)
Fig. 12. Scenarios of a two fish interaction, (a) face to face, (b) crossing and (c) chasing. 
correctly followed by the proposed tracking scheme. An exception was that fish $\mathrm{B}^{\prime}$ and fish A were the bipartite nearest neighbor of each other and fish $A$ and $B^{\prime}$ would be incorrectly paired. On the other hand, this exception case seldom occurred in the actual observations. Fig. 11(c) presents a common interaction in the chasing behavior, in which fish A followed the movement of fish B. Fish $A^{\prime}$ and fish $B$ formed a nearest pair; however, fish $A^{\prime}$ and fish $B$ were not allowed to be paired because fish $\mathrm{A}^{\prime}$ did not appear within the tracking gate of fish $\mathrm{B}$ according to the pairing constraints. Therefore, the proposed tracking scheme could accurately follow the correct trajectories by applying the pose constraint; even the fish moved closely at a high speed.

Moreover, the behavior of backwards swimming was observed occasionally when a fish ran into the boundary or aggregated with other fish. The fish moved backward slightly, probably due to the water flow produced by the other fish. The slight backward movements were also tracked by the proposed optimization data association scheme. The proposed tracking system could be also applied to observe the behavior of other types of Danios, such as Pearl Danio, Leopard Danio, etc., because Danios show cone shapes in the top view and provide a similar appearance and shape features to the tracking system. The proposed method could recognize the head and tail of these fish and acquire the movement trajectories.

Occlusions and swapping of tracking targets are common issues suffered in multiple objects tracking. In particular, in fish tracking, individuals present similar visual features (e.g., shape and appearance) and are difficult to identify from each other. In this study, the water depth was limited to minimize the occlusions among fish. The maximal occlusion rate was reduced to $4.072 \%$ with 5 individuals while the overall tracking error for 5 individuals was only $1.572 \%$. The overall tracking error proved the excellent ability of this work in tracking individuals from occlusions. A previous study showed that the fish behaviors in shallow water could efficiently reflect the effects under chemical stress (Quach et al., 2013). Controlling the water depth is an alternative solution for two-dimensional behavioral monitoring in practical applications with a low cost at the current stage (Qian et al., 2014). A bottom light source was installed in the monitoring system to eliminate the reflection from the water surface and shadows. A uniform surface light panel consisting of a light emitting diode (LED) array was adopted as the light source to provide stable illumination and enhance the contrast of the fish images. Light noises were depressed significantly from the observation images. In addition, a region of interest (ROI) was set manually to remove the disturbance from the bottom edges (Fig. 2b).

Tracking errors were caused mainly by occlusions or the attaching of fish when individuals aggregated. The tracker presented in this paper was not designed to segment the individual fish from the attached fish images. Therefore, the tracker could not deal with the attached fish during occlusion. Instead, it would keep the current position until the occlusion ended and tried to catch the tracking targets again according to the previous location and posture. During this process, the tracker might not follow the correct individual and the individual trajectories could be swapped due to the lack of ability to identify each individual. The recent developed individual fish identification algorithm could be a solution for accurate fish tracking from occlusions (Pérez-Escudero et al., 2014; Qian et al., 2014). Integration of this appearance identification method and the proposed tracking scheme could be considered to improve the robustness of multiple tracking. On the other hand, this fish identification method is computationally expensive. A computationally inexpensive method should be studied in further work. In addition, the model-based tracking provides an alternative solution to identifying individual fish from occlusions (Xia et al., 2014).

This paper presented a reliable posture-based individual tracking method that was evaluated under the controlled illumination in laboratory conditions. The presented observation system could be applied to in situ behavioral observation by integrating water-cycling system. Furthermore, the in situ observation systems can be extended to a largescale sensing network in the future.

\section{Conclusions}

A multiple fish tracking scheme was presented by integrating the movement direction of individual fish and the global nearest neighbor. Automatic initialization for fish tracking was implemented by extracting the individual fish from the background. In addition, identification of the fish head and tail was developed based on the gray features of the head and tail images. The movement direction of the individual fish was obtained accordingly and was integrated to the tracking system. The remarkable tracking performance of the proposed method was achieved through behavioral observations of 2-5 individuals. Posture analysis and group behaviors of the multiple individuals were examined further to reveal the movement patterns of fish. The advantages of the posture tracking scheme were evaluated by the tracking experiments on multiple fish. The proposed multiple fish tracking could be an efficient and fundamental tool for modern biological and ecological studies. Multiple fish tracking could be the key technique for building a sensing network in aquatic ecosystems and be applied to long-term environmental monitoring.

\section{Acknowledgments}

This study was supported by the National Natural Science Foundation of China (Grant No. 41406112 and 41501538). Guangdong Provincial Department of Science and Technology (2015A020216011, 2016A020221016), and Guangzhou Science Technology and Innovation Commission (201510010294).

\section{References}

Bae, M.J., Park, Y.S., 2014. Biological early warning system based on the responses of aquatic organisms to disturbances: a review. Sci. Total Environ. 466, 635-649.

Barry, M.J., 2012. Application of a novel open-source program for measuring the effects of toxicants on the swimming behavior of large groups of unmarked fish. Chemosphere $86(9), 938-944$

Branson, K., Robie, A.A., Bender, J., Perona, P., Dickinson, M.H., 2009. High-throughput ethomics in large groups of Drosophila. Nat. Methods 6 (6), 451-457.

Cai, Y., de Freitas, N., Little, I.I., 2006. Robust Visual Tracking for Multiple Targets. Springer, Berlin Heidelberg, pp. 107-118.

Chon, T.S., Chung, N., Kwak, I.S., Kim, J.S., Koh, S.C., Lee, S.K., ... Cha, E.Y., 2005. Movement behaviour of medaka (Oryzias latipes) in response to sublethal treatments of diazinon and cholinesterase activity in semi-natural conditions. Environ. Monit. Assess. 101 (1-3), 1-21.

Delcourt, J., Beco, C., Ylieff, M.Y., Caps, H., Vandewalle, N., Poncin, P., 2006. Comparing the EthoVision 2.3 system and a new computerized multitracking prototype system to measure the swimming behavior in fry fish. Behav. Res. Methods 38 (4), 704-710.

Delcourt, J., Becco, C., Vandewalle, N., Poncin, P., 2009. A video multitracking system for quantification of individual behavior in a large fish shoal: advantages and limits. Behav. Res. Methods 41 (1), 228-235.

Delcourt, J., Denoël, M., Ylieff, M., Poncin, P., 2013. Video multitracking of fish behaviour: a synthesis and future perspectives. Fish Fish. 14 (2), 186-204.

Fontaine, E., Lentink, D., Kranenbarg, S., Müller, U.K., van Leeuwen, J.L., Barr, A.H., Burdick J.W., 2008. Automated visual tracking for studying the ontogeny of zebrafish swimming. J. Exp. Biol. 211 (8), 1305-1316.

Gerhardt, A., 1999. Recent trends in online biomonitoring for water quality control. In: Gerhardt, A. (Ed.), Biomonitoring of Polluted Water-Reviews on Actual Topics. Environ. Res. Forum Vol. 9. Zurich, Switzerland, TTP, pp. 95-119.

Gerlai, R., Lee, V., Blaser, R., 2006. Effects of acute and chronic ethanol exposure on the behavior of adult zebrafish (Danio rerio). Pharmacol. Biochem. Behav. 85 (4), 752-761.

Harville, M., 2002. A framework for high-level feedback to adaptive, per-pixel, mixtureof-gaussian background models. Computer Vision-ECCV 2002. Springer, Berlin Heidelberg, pp. 543-560.

Kane, A.S., Salierno, J.D., Gipson, G.T., Molteno, T.C., Hunter, C., 2004. A video-based movement analysis system to quantify behavioral stress responses of fish. Water Res. 38 (18), 3993-4001.

Kato, S., Tamada, K., Shimada, Y., Chujo, T., 1996. A quantification of goldfish behavior by an image processing system. Behav. Brain Res. 80 (1), 51-55.

Kato, S., Nakagawa, T., Ohkawa, M., Muramoto, K., Oyama, O., Watanabe, A., ... Sugitani, K, 2004. A computer image processing system for quantification of zebrafish behavior. J. Neurosci. Methods 134 (1), 1-7.

Konstantinova, P., Udvarev, A., Semerdjiev, T., 2003. A study of a target tracking algorithm using global nearest neighbor approach. Proceedings of the International Conference on Computer Systems and Technologies (CompSysTech'03) (June).

Kuklina, I., Kouba, A., Kozák, P., 2013. Real-time monitoring of water quality using fish and crayfish as bio-indicators: a review. Environ. Monit. Assess. 185 (6), 5043-5053. 
ARTICLE IN PRESS

C. Xia et al. / Ecological Informatics $x x x$ (2016) $x x x-x x x$

9

Liu, Y., Lee, S.-H., Chon, T.-S., 2011. Analysis of behavioral changes of zebrafish (Danio rerio) in response to formaldehyde using self-organizing map and a hidden Markov model. Ecol. Model.

Ma, H., Tsai, T.F., Liu, C.C., 2010. Real-time monitoring of water quality using temporal trajectory of live fish. Expert Syst. Appl. 37 (7), 5158-5171.

Masud, S., Singh, I.J., Ram, R.N., 2005. Behavioural and hematological responses of Cyprinus carpio exposed to mercurial chloride. J. Environ. Biol. 26, 393-397.

Noldus, L.P., Spink, A.I., Tegelenbosch, R.A., 2001. EthoVision: a versatile video tracking system for automation of behavioral experiments. Behav. Res. Methods Instrum. Comput. 33 (3), 398-414.

Papadakis, V.M., Papadakis, I.E., Lamprianidou, F., Glaropoulos, A., Kentouri, M., 2012. A computer-vision system and methodology for the analysis of fish behavior. Aquac. Eng. 46, 53-59.

Park, Y.S., Chung, N.I., Choi, K.H., Cha, E.Y., Lee, S.K., Chon, T.S., 2005. Computational characterization of behavioral response of medaka (Oryzias latipe) treated with diazinon. Aquat. Toxicol. 71 (3), 215-228.

Pérez-Escudero, A., Vicente-Page, I., Hinz, R.C., Arganda, S., de Polavieja, G.G., 2014 idTracker: tracking individuals in a group by automatic identification of unmarked animals. Nature Methods.

Pinkiewicz, T., Williams, R., Purser, J., 2008. Application of the particle filter to tracking of fish in aquaculture research. Digital Image Computing: Techniques and Applications (DICTA), 2008. IEEE, pp. 457-464 (December).
Qian, Z.M., Cheng, X.E., Chen, Y.Q., 2014. Automatically detect and track multiple fish swimming in shallow water with frequent occlusion. PLoS One 9 (9), e106506.

Quach, Q.K., Chon, T.S., Kim, H., Van Nguyen, T., 2013. One and two-individual movements of fish after chemical exposure. J. Korean Phys. Soc. 63 (1), 18-27.

Ren, Z., Wang, Z., 2010. Differences in the behavior characteristics between Daphnia magna and Japanese madaka in an on-line biomonitoring system. J. Environ. Sci. 22 (5), 703-708

Sauvola, I., Pietikainen, M., 2000. Adaptive document image binarization. Pattern Recogn. 33 (2), 225-236.

Suzuki, K., Takagi, T., Hiraishi, T., 2003. Video analysis of fish schooling behavior in finite space using a mathematical model. Fish. Res. 60 (1), 3-10.

Swain, H.A., Sigstad, C., Scalzo, F.M., 2004. Effects of dizocilpine (MK-801) on circling behavior, swimming activity, and place preference in zebrafish (Danio rerio). Neurotoxicol. Teratol. 26 (6), 725-729.

Wang, L., Ren, Z., Kim, H., Xia, C., Fu, R., Chon, T.S., 2014. Characterizing response behavior of medaka (Oryzias latipes) under chemical stress based on self-organizing map and filtering by integration. Ecol. Inf. 29, 107-118.

Xia, C., Li, Y., Lee, J.M., 2014. A visual measurement of fish locomotion based on deformable models. Intelligent Robotics and Applications. Springer International Publishing, pp. 110-116.

Please cite this article as: Xia, C., et al., Posture tracking of multiple individual fish for behavioral monitoring with visual sensors, Ecological Informatics (2016), http://dx.doi.org/10.1016/j.ecoinf.2016.07.004 